\title{
INDEX TO VOLUME 80
}

\section{RESEARCH ANNOUNCEMENTS}

Aarnes, Johan F. Frobenius reciprocity of differentiable representations, 337

Adler, Andrew and Williams, R. Douglas. Restricted ideals in rings of analytic functions, 169

Ahmad, Shair. Periodically perturbed conservative systems, 122

Alexander, J. P., Conner, P. E., Hamrick, G. C. and Vick, J. W. Witt classes of integral representations of an Abelian p-group, 1179

Alexander, J. P., Hamrick, G. C. and Vick, J. W. Bilinear forms and cyclic group actions, 730

Allenby, R. B. J. T. and Tang, C. Y. On the Frattini subgroups of generalized free products, 119

de Andrade, Rubens Leão. Complete convex hypersurfaces of a Hilbert space, 576

Anh, Nguyen Huu. Algebraic groups with square-integrable representations, 539

Anshel, Michael and Stebe, Peter. The solvability of the conjugacy problem for certain HNN groups, 266

Aron, Richard M. and Schottenloher, Martin. Compact holomorphic mappings on Banach spaces and the approximation property, 1245

Azoff, Edward A. and Gilfeather, Frank. Measurable choice and the invariant subspace problem, 893

Bak, Anthony. The computation of surgery groups of odd torsion groups, 1113

Ball, Joseph A. Factorization and invariant subspaces for noncontractions, 896

Barbosa, J. L. and do Carmo, M. Stable minimal surfaces, 581

Barrow, David L. See Stroud, A. H.

Beale, J. Thomas and Rosencrans, Steven I. Acoustic boundary conditions, 1276

Beck, Anatole. Conditional independence, 1169

Berenstein, C. A. and Dostal, M. A. A lower estimate for exponential sums, 687

Berger, M. S. and Podolak, E. On nonlinear Fredholm operator equations, 861

Bernau, S. J. Lateral completion for arbitrary lattice groups, 334

Bers, Lipman. On spaces of Riemann surfaces with nodes, 1219

Bichteler, Klaus. On sequences of measures, 839

Billera, Louis J. and Bixby, Robert E. Market representations of n-person games, 522 Bixby, Robert E. The minimum number of edges and vertices in a graph with edge connectivity $n$ and $m$-bounds, 700

, See Billera, Louis $\mathrm{J}$.

Blass, J. On the Diophantine equation $Y^{2}+K=X^{5}, 329$

Bloch, S. Torsion algebraic cycles, $K_{2}$ and Brauer groups of function fields, 941

Block, Richard E. Irreducible representations of Lie algebra extensions, 868

de Boor, Carl. A remark concerning perfect splines, 724

Bouldin, Richard. Positive near-approximants and some problems of Halmos, 313

Bourgin, D. G. A degree for nonacyclic multiple-valued transformations, 59

Brayton, R. K., Coppersmith, Donald and Hoffman, A. J. Self-orthogonal latin squares of all orders $n \neq 2,3,6,116$

Brézis, Haim and Browder, Felix E. Some new results about Hammerstein equations, 567

Brooks, James K. and Dinculeanu, Nicolae. Lebesgue spaces for bilinear vector integration theory, 821 
Browder, Felix E. See Brézis, Haim

Brown, Edgar H., Jr. A product formula for an Arf-Kervaire invariant, 873

Brown, Robert F. Fixed points of endomorphisms of compact groups, 293

Byrnes, Christopher I. A spectral decomposition theorem for certain harmonic algebras, 1271

Cannon, John R., Henry, Daniel B. and Kotlow, Daniel B. Continuous differentiability of the free boundary for weak solutions of the Stefan problem, 45

Cantor, M. Sobolev inequalities for Riemannian bundles, 239

Cappell, Sylvain E. Manifolds with fundamental group a generalized free product. I, 1193

- Unitary nilpotent groups and Hermitian K-theory. I, 1117

Carey, R. W. and Pincus, J. D. Perturbation by trace class operators, 758

Carlitz, L., Scoville, Richard and Vaughan, Theresa. Enumeration of pairs of permutations and sequences, 881

do Carmo, M. See Barbosa, J. L.

Casselman, W. On a padic vanishing theorem of Garland, 1001

Cattani, Eduardo H. On the partial compactification of the arithmetic quotient of a period, matrix domain, 330

Cenzer, Douglas. Inductively defined sets of reals, 485

Cesari, Lamberto. A necessary and sufficient condition for lower semicontinuity, 467

Cesari, L. and Kaiser, P. Closed operators and existence theorems in multidimensional problems of the calculus of variations, 473

Chafee, Nathaniel and Infante, E. F. Bifurcation and stability for a nonlinear parabolic partial differential equation, 49

Chatterji, S. D. A subsequence principle in probability theory (applied to the law of the iterated logarithm), 495

Chen, Kuo-Tsai. Solvability on manifolds by quadratures permitting only integrals, 1210

Chen, Su-Shing. On Lobatchewsky manifolds, 244

Chewning, W. C. A dynamical system on $E^{4}$ neither isomorphic nor equivalent to a differential system, 150

Choi, M. D. and Davis, Chandler. The spectral mapping theorem for joint approximate point spectrum, 317

Chou, Ching. Weakly almost periodic functions with zero mean, 297

Cobb, Peter V. Z. $P_{n}$-spaces and $n$-fold loop spaces, 910

Cochran, James Alan. Square-integrable kernels and growth estimates for their singular values, 661

Cohn, Leslie. Some theorems on C-functions, 921

Coifman, Ronald R. and Weiss, Guido. Central multiplier theorems for compact Lie groups, 124

Conner, P. E. See Alexander, J. P., Hamrick, G. C. and Vick, J. W.

Coppersmith, Donald. See Brayton, R. K. and Hoffman, A. J.

Curtis, D. W. and Schori, R. M. $2^{X}$ and $C(X)$ are homeomorphic to the Hilbert cube, 927

Dahmen, Wolfgang and Görlich, Ernst. A conjecture of M. Golomb on optimal and nearly. optimal linear approximation, 1199

Danskin, John M. Values in differential games, 449

Davis, Chandler. See Choi, M. D.

Dennis, R. Keith and Stein, Michael R. Injective stability for $K_{2}$ of local rings, 1010

Dinculeanu, Nicolae. See Brooks, James K.

Dodziuk, Jozef. Combinatorial and continuous Hodge theories, 1014

Dostal, M. A. See Berenstein, C. A.

Drasin, David. A meromorphic function with assigned Nevanlinna deficiencies, 766 
Drucker, Daniel. Orbit structure of the exceptional Hermitian symmetric spaces. I, 285

- Orbit structure of the exceptional Hermitian symmetric spaces. II, 1225

Drury, S. W. The Fatou-Zygmund property for Sidon sets, 535.

Dyer, M. N. Homotopy trees for periodic groups, 1279

Eklund, Neil. Generalized super-parabolic functions, 343

Ellis, Richard S. and Pinsky, Mark A. Asymptotic nonuniqueness of the Navier-Stokes equations in kinetic theory, 1160

Erdelyi, I. Unbounded operators with spectral capacities, 1108

Ewing, John. Some examples of sphere bundles over spheres which are loop spaces mod $p$, 935

Faires, Barbara. On Vitali-Hahn-Saks type theorems, 670

Farias, Antonio O. Immersions of the circle and extensions to orientation-preserving map. pings, 578

Feder, S. and Gitler, S. Stunted projective spaces and the J-order of the Hopf bundle, 748

Fefferman, Charles. On the Bergman kernel and biholomorphic mappings of pseudoconvex domains, 667

Fein, Burton and Schacher, Murray. Finite subgroups of finite dimensional division algebras, 290

Fenichel, Neil. Asymptotic stability with rate conditions for dynamical systems, 346

Fischer, Arthur E. and Marsden, Jerrold E. Manifolds of Riemannian metrics with prescribed scalar curvature, 479

Fischer, Arthur E. and Wolf, Joseph A. The Calabi construction for compact Ricci flat Riemannian manifolds, 92

Fisher, Michael J. Properties of three algebras related to $L_{p^{-} \text {-multipliers, } 262}$

Flanigan, F. J. Radical embedding, genus, and toroidal derivations of nilpotent associative algebras, 986

Folland, G. B. and Stein, E. M. Parametrices and estimates for the $\bar{\partial}_{b}$ complex on strongly pseudoconvex boundaries, 253

Francis, George K. Branched and folded parametrizations of the sphere, 72

Friedland, Shmuel. Nonoscillation and integral inequalities, 715

Geymonat, Giuseppe and Grubb, Gerd. Special theory for boundary value problems for elliptic systems of mixed order, 1255

Giffen, Charles H. Schur homotopy of the simplest group, 957

Gilbert, John E. $L^{p}$-convolution operators and tensor products of Banach spaces, 1127

Gilfeather, Frank. See Azoff, Edward A.

Gitler, S. See Feder, S.

Goelman, Don. Rational critical points of the reduced norm of an algebra, 138

Goldberg, Samuel I. and Ishihara, Toru. Harmonic quasiconformal mappings of Riemannian manifolds, 562

Goldberg, Wallace. On the determination of a Hill's equation from its spectrum, 1111

Goldsmith, Deborah Louise. Motions of links in the 3-sphere, 62

Görlich, Ernst. See Dahmen, Wolfgang

Gray, Hohn W. Quasi-Kan extensions for 2-categories, 142

Greenspan, Donald. A physically consistent, discrete $n$-body model, 553

Grosshans, Frank. Open sets of points with good stabilizers, 518

Grubb, Gerd. See Geymonat, Giuseppe

Gudder, S. and Strawther, D. Orthogonality and nonlinear functionals, 946

Guerra, F., Rosen, L. and Simon, B. The pressure is independent of the boundary conditions for $P(\phi)_{2}$ field theories, 1205

Gupta, Ram Prakash. On decompositions of a multi-graph into spanning subgraphs, 500

Guthrie, J. A. and Henry, Michael. Metrization and paracompactness in terms of real functions, 720 
Halpern, J. D. and Howard, Paul E. Cardinal addition and the axiom of choice, 584

Hamrick, G. C. See Alexander, J. P., Conner, P. E. and Vick, J. W.

- See Alexander, J. P. and Vick, J. W.

Hardt, Robert M. Homology and images of semianalytic sets, 675

Hartshorne, Robin, Rees, Elmer and Thomas, Emery. Nonsmoothing of algebraic cycles on Grassmann varieties, 847

Harvey, F. Reese and Lawson, H. Blaine, Jr. Boundaries of complex analytic varieties, 180

Hebert, D. J., Jr. Nonlinear parabolic equations and probability, 965

Hedrick, Mark Blondeau. The permanent at a minimum on certain classes of doubly stochastic matrices, 836

Heidel, J. W. See Stafford, R. A.

Hejhal, Dennis A. A remark on the Lindelöf hypothesis, 695

Hemion, Geoffrey. See McPherson, M.

Henry, Daniel B. See Kotlow, Daniel B, and Cannon, John R.

Henry, Michael. See Guthrie, J. A.

Hilden, Hugh M. Every closed orientable 3-manifold is a 3-fold branched covering space of $S^{3}, 1243$

Hochster, Melvin. The equicharacteristic case of some homological conjectures on local rings, 683

Hochster, Melvin and Roberts, Joel L. Actions of reductive groups on regular rings and Cohen-Macaulay rings, 281

Hodgson, J. P. E. Subcomplexes of Poincaré complexes, 1146

Hoffman, A. J. See Brayton, R. K. and Coppersmith, Donald

Holme, Audun. An embedding-obstruction for projective varieties, 932

Holmes, Richard, Scranton, Bruce and Ward, Joseph. Best approximation by compact operators. II, 98

Horowitz, Charles. Zeros of functions in the Bergman spaces, 713

Howard, Paul E. See Halpern, J. D.

Hsieh, M. S. See Morrell, J. S. and Retherford, J. R.

Hunt, Richard A. and Young, Wo-Sang. A weighted norm inequality for Fourier series, 274

Husseini, S. Y. Manifolds with the fixed point property. I, 664

Hutton, C. V., Morrell, J. S. and Retherford, J. R. Approximation numbers and Kolmogoroff diameters of bounded linear operators, 462

Infante, E. F. See Chafee, Nathaniel

Ishihara, Toru. See Goldberg, Samuel I.

James, Robert C. A separable somewhat reflexive Banach space with nonseparable dual, 738

Jayne, J. E. The space of class $\alpha$ Baire functions, 1151

Kaigh, W. D. A conditional local limit theorem and its application to random walk, 769

Kaiser, P. See Cesari, L.

Kauffman, Louis H. Products of knots, 1104

Kiernan, Peter. On the compactifications of arithmetic quotients of symmetric spaces, 109

Kiremidjian, Garo K. On the extension of boundary integrable almost complex structure, 982

Knight, William J. Existence of solutions of differential equations in Banach space, 148

Koliha, J. J. Pseudo-inverses of operators, 325

Koschorke, Ulrich. Singularities and bordism of q-plane fields and of foliations, 760

Kotlow, Daniel B. See Cannon, John R. and Henry, Daniel B.

Kreith, Kurt. Nonselfadjoint fourth order differential equations with conjugate points, 1190

Kronstadt, Eric P. General interpolating sequences in disks and polydisks, 132

Kuelbs, J. An inequality for the distribution of a sum of certain Banach space valued random variables, 549 
Kulshrestha, P. K. Coefficients for alpha-convex univalent functions, 341

Kuo, Hui-Hsiung. The Morse-Palais lemma on Banach spaces, 363

Laha, R. G. A class of square-integrable irreducible unitary representations of some linear groups over commutative p-fields, 718

Lawson, H. Blaine, Jr. See Harvey, F. Reese

Leger, G. and Luks, E. Cohomology and weight systems for nilpotent Lie algebras, 77

Lehrer, G. I. Weil representations and cusp forms on unitary groups, 1137

Levinson, Norman. Zeros of derivative of Riemann's $\xi$-function, 951

Lim, Teck-Cheong. A fixed point theorem for multivalued nonexpansive mappings in a uniformly convex Banach space, 1123

Lin, James P. H-spaces with finitely generated cohomology algebras, 1233

Lins Neto, Alcides. Generalized gradient fields and electrical circuits, 991

Longstaff, W. E. Strongly reflexive lattices, 875

Loos, Ottmar. A structure theory of Jordan pairs, 67

Lucas, Dean. Properties of rank preserving weak maps, 127

Luks, E. See Leger, G.

MacBain, John. Global bifurcation theorems for noncompact operators, 1005

MacDonald, John L. Conditions for a universal mapping of algebras to be a monomorphism, 888

Madsen, I. and Milgram, R. J. The oriented topological and PL cobordism rings, 855

Mah, P. and Naimpally, S. A. Open and uniformly open relations, 1157

Mane, Ricardo. Persistent manifolds are normally hyperbolic, 90

Merrero, Osvaldo. Completion and embedding between pseudo $(v, k, \lambda)$-designs and $(v, k, \lambda)$-designs, 103

Marsden, J. A formula for the solution of the Navier-Stokes equations based on a method of Chorin, 154

Marsden, Jerrold E. See Fischer, Arthur E.

Marsden, M. J. Quadratic spline interpolation, 903

Martio, O. and Srebro, U. Automorphic mappings in $R^{n}, 692$

Maskit, Bernard. Moduli of marked Riemann surfaces, 773

Mather, John N. Simplicity of certain groups of diffeomorphisms, 271

May, Robert D. Stability and transversality, 85

McPherson, M. and Hemion, Geoffrey. Locally prime arcs with finite penetration index, 531

Mesirov-Kazdan, Jill P. Calculus of variations: Perturbations preserving condition (C), 1260

Metzler, Richard C. Representation of partially ordered linear algebras, 939

Meyer, R. E. Exponential action of a pendulum, 164

Milgram, R. J. See Madsen, I.

Minda, Carl David. Extremal length, reproducing differentials and Abel's theorem, 735

Montesinos, José M. A representation of close, orientable 3-manifolds as 3-fold branched coverings of $S^{3}, 845$

Morrell, J. S., Hsieh, M. S. and Retherford, J. R. On ideals of compact operators, 907

, See Hutton, C. V. and Retherford, J. R.

Mosco, Umberto and Strang, Gilbert. One-sided approximation and variational inequalities, 308

Myers, Sally Ellene. A boundary maximum principle for degenerate elliptic-parabolic inequalities, for characteristic boundary points, 527

Nagel, Alexander, Rivière, Nestor and Wainger, Stephen. On Hilbert transforms along curves, 106

Naimpally, S. A. See Mah, P.

Nashed, M. Z. and Votruba, G. F. A unified approach to generalized inverses of linear operators: I. Algebraic, topological, and projectional properties, 825

, A unified approach to generalized inverses of linear operators: II. Extremal and proximinal properties, 831 
Nashed, M. Z. and Wahba, Grace. Regularization and approximation of linear operator equations in reproducing kernel spaces, 1213

Neumann, Walter D. Cyclic suspension of knots and periodicity of signature for singularities, 977

Nishimura, Jun-ichi. See Suzuki, Satoshi

Osborne, M. S. Lie algebra cohomology of certain infinite-dimensional representations, 852

Osher, Stanley. An ill-posed problem for a strictly hyperbolic equation in two unknowns near a corner, 705

Palis, J. Vector fields generate few diffeomorphisms, 503

Paré, Robert. Colimits in topoi, 556

Passow, Eli and Raymon, Louis. Restricted approximation and interpolation, 750

Pearcy, Carl and Salinas, Norberto. Finite-dimensional representations of separable $C^{*}$. algebras, 970

Pedersen, Erik Kjaer. Topological concordances, 658

Petrie, Ted. Obstructions to transversality for compact Lie groups, 1133

Pfaltzgraff, J. A. Quasiconformal extension of holomorphic mappings of a ball in $C^{n}, 543$ Phillips, Anthony. Smooth maps of constant rank, 513

Pincus, J. D. See Carey, R. W.

Pinsky, Mark A. See Ellis, Richard S.

Pless, Vera and Sloane, N. J. A. Binary self-dual codes of length 24, 1173

Podolak, E. See Berger, M. S.

Poénaru, V. A remark on simply-connected 3-manifolds, 1203

Pontes, Edmilson. Isometric minimal immersions of $S^{3}(a)$ in $S^{N}(1), 1239$

Pukanszky, L. Characters of connected Lie groups, 709

Randell, Richard C. Generalized Brieskorn manifolds, 111

Randol, Burton. Small eigenvalues of the Laplace operator on compact Riemann surfaces, 996

Ravenel, Douglas C. and Wilson, W. Stephen. The Hopf ring for complex cobordism, 1185 Raymon, Louis. See Passow, Eli

Reed, G. M. and Zenor, P. L. Preimages of metric spaces, 879

Rees, Elmer. See Hartshorne, Robin and Thomas, Emery

Retherford, J. R. See Hutton, C. V. and Morrell, J. S.

, See Hsieh, M. S. and Morrell, J. S.

Rice, John R. Adaptive quadrature: Convergence of parallel and sequential algorithms, 1250

Rice, Michael D. Covering and function theoretic properties of uniform spaces, 159

Rivière, Nestor. See Nagel, Alexander and Wainger, Stephen

Roberts, Joel L. See Hochster, Melvin

Robinson, C. A. Stable homotopy theory over a fixed base space, 248

Rosen, L. See Guerra, F. and Simon, B.

Rosencrans, Steven I. See Beale, J. Thomas

Rosenkrantz, Walter A. A convergent family of diffusion processes whose diffusion coefficients diverge, 973

Sacker, Robert J. and Sell, George R. A note on Anosov diffeomorphisms, 278

Salinas, Norberto. See Pearcy, Carl

Sario, Leo. A criterion for the existence of biharmonic Green's functions, 1183

Schacher, Murray. See Fein, Burton

Schlesinger, James W. Sets of colorings of circuits, 721

Schori, R. M. See Curtis, D. W.

Schottenloher, Martin. See Aron, Richard M.

Schultz, Reinhard. Differentiable $Z_{p}$ actions on homotopy spheres, 961

Scoville, Richard. See Carlitz, L. and Vaughan, Theresa

Scranton, Bruce, See Holmes, Richard and Ward, Joseph 
Seabury, Chester. Some extension theorems for regular maps of Stein manifolds, 1223

Seidman, Thomas I. A well-posed problem for the heat equation, 901

Sell, George R. See Sacker, Robert J.

Sharpley, Robert. Interpolation of operators for $\Lambda$ spaces, 259

Simoes, Plinio. On a class of minimal cones in $R^{n}, 488$

Simon, B. See Guerra, F. and Rosen, L.

Singer, Ivan. On the extension of basic sequences to bases, 771

Sloane, N. J. A. See Pless, Vera

Soare, Robert I. Automorphisms of the lattice of recursively enumerable sets, 53

Srebro, U. See Martio, O.

Strafford, R. A. and Heidel, J. W. A new comparison theorem for scalar Riccati equations, 754

Stebe, Peter. See Anshel, Michael

Stefan, P. Accessibility and foliations with singularities, 1142

Stein, E. M. See Folland, G. B.

Stein, Junior. Hilbert space and variational methods for singular selfadjoint systems of differential equations, 744

Stein, Michael R. See Dennis, R. Keith

Stein, S. K. Tiling space by congruent polyhedra, 819

Stevenson, James O. Holomorphy of composition, 300

Strang, Gilbert. See Mosco, Umberto

Strawther, D. See Gudder, S.

Stroud, A. H. and Barrow, David L. Gauss formulas for the Dirichlet problem, 1230

Sussmann, Héctor J. On quotients of manifolds: A generalization of the closed subgroup theorem, 573

Sutherland, Colin E. Direct integral theory for weights, and the Plancherel formula, 456

Suzuki, Satoshi and Nishimura, Jun-ichi. Higher differential algebras of discrete valuation rings, 865

Tang, C. Y. See Allenby, R. B. J. T.

Tapia, R. A. A general approach to Newton's method for Banach space problems with equality constraints, 355

Taussky, Olga. Additive commutators between $2 \times 2$ integral matrix representations of orders in identical or different quadratic number fields, 885

Thomas, Emery. See Hartshorne, Robin and Rees, Elmer

Thomson, James. The commutant of analytic Toeplitz operators, 915

Thurston, William. Foliations and groups of diffeomorphisms, 304

Turner, Stuart. The zeta function of a class of rational varieties, 917

Vaseekaran, C. Equivariant homotopy theory, 322

Vaughan, Theresa. See Carlitz, L. and Scoville, Richard

Vick, J. W. See Alexander, J. P. and Hamrick, G. C.

-, See Alexander, J. P., Conner, P. E. and Hamrick, G. C.

Volk, B. On the maximum Nth diameter, 446

Votruba, G. F. See Nashed, M. Z.

Výborny, R. The Hadamard three-circles theorems for partial differential equations, 81

Wahba, Grace. See Nashed, M. Z.

Wainger, Stephen. See Nagel, Alexander and Rivière, Nestor

Walsh, Bertram. An approximation property characterizes ordered vector spaces with lattice-ordered duals, 1165

Ward, Joseph. See Holmes, Richard and Scranton, Bruce

Weiss, Guido. See Coifman, Ronald R.

White, James H. Minimal total absolute curvature for orientable surfaces with boundary, 361 
Whitten, Wilbur. Characterizations of knots and links, 1265

Williams, R. Douglas. See Adler, Andrew

Wilson, W. Stephen. See Ravenel, Douglas C.

Winkler, William. Some results in Doeblin's theory of Markov chains, 728

Winter, David J. Normal field extensions $K / k$ and $K / k$-bialgebras, 506

Wolf, Joseph A. See Fischer, Arthur E.

Wong, James S. W. Positive definite functions and Volterra integral equations, 679

Wong, Kai-Tak. On extended structures of a closed operator related to semigroup theory and the abstract Cauchy problem, 350

Young, Wo-Sang. On rearrangements of Walsh-Fourier series and Hardy-Littlewood type maximal inequalities, 490 See Hunt, Richard A.

$\mathrm{Yu}$, Chung-Ling. The existence for the solution of the elliptic Cauchy problem, 545

Zassenhaus, Hans. Reduction theory in algebraic number fields, 955

Zehnder, E. An implicit function theorem for small divisor problems, 174

Zenor, P. L. See Reed, G. M.

Zettl, Anton. A characterization of the factors of ordinary linear differential operators, 498

\section{INVITED ADDRESSES}

Andrews, George E., A general theory of identities of the Rogers-Ramanujan type, 1033 Berlekamp, Elwyn R., 652

Bharucha-Reid, A. T., 816

Bishop, Errett A., 440

Browder, Felix E., 440

Dudley, Richard M., 440

Duffin, R. J., Some problems of mathematics and science, 1053

$\longrightarrow, 652$

Fitzpatrick, Ben, Jr., 445

Fleming, Wendell H., 440

Gallagher, Patrick X., 440

Garsia, Adriano M., 652

Gluck, Herman R., 440

Hall, Marshall, Jr., 238

Hamstrom, Mary-Elizabeth, Homotopy in homeomorphism spaces, TOP and PL, 207

Hartshorne, Robin, Varieties of small codimension in projective space, 1017

$\longrightarrow, 440$

Harvey, Reese, Three structure theorems in several complex variables, 633

$\longrightarrow, 440$

Hill, Paul D., 445

Hirzebruch, F. E. P., 440

Jacobson, Nathan, 652

Kahane, J.-P., Best approximation in $L^{1}(\mathrm{~T}), 788$

$\longrightarrow, 444$

Karp, R., 441

Knopp, Marvin I., Some new results on the Eichler cohomology of automorphic forms, 607

Kobayashi, Shoshichi, 652

Kuratowski, Kazimierz, 238

Lang, Serge, Higher dimensional diophantine problems, 779

Lawson, H. Blaine, Jr., Foliations, 369

Lichtenbaum, Stephen, 443 
Lieb, Elliott H., 443

Matsusaka, T., Global deformation of polarized varieties, 1

Meyer, A., 441

Mitchell, Barry M., 652

Mycielski, Jan, 440

Nashed, M. Zuhair, 445

Nirenberg, Louis, 652

Ogg, Andrew P., 651

Osofsky, Barbara L., The subscript of $\aleph_{n}$, projective dimension and the vanishing of $\lim (n), 8$

Petryshyn, W. Y., 440

Phelps, Robert R., 651

Ray-Chaudhuri, Dijen K., 653

Raymond, Frank A., 444

Richards, J. Ian, On the incompatibility of two conjectures concerning primes; a discussion of the use of computers in attacking a theoretical problem, 419 , 441

Rodin, Burton, The method of extremal length, 587

Samuelson, Paul A., 652

Schwartz, J. T., Semantic and syntactic issues in programming, 185

$\longrightarrow, 441$

Shoenfield, J. R., 816

Shub, M., Dynamical systems, filtrations and entropy, 27

Simmons, James, 441

Solomon, Louis, 653

Stark, Harold M., 441

Thompson, John G., 652

Tulcea, A. Ionescu, On measurability, pointwise convergence and compactness, 231

Weinstein, Alan D., 653

\section{BOOK REVIEWS}

Dixon, John D. Review of Infinite linear groups by B. A. F. Wehrfritz, 1071

Kline, Morris. See Rota, Gian-Carlo

Rota, Gian-Carlo. Review of Mathematical thought from ancient to modern times by Morris Kline, 805

Wehrfritz, B. A. F. See Dixon, John D.

\section{MISCELLANEOUS}

Albert, Abraham Adrian. See Jacobson, Nathan

Board of Trustees, 1973, 184

Council Members, 1973, 184

Election results, 1973,368

Index to Volume 80,1284

Jacobson, Nathan. Obituary of Abraham Adrian Albert, 1905-1972, 1075

Lehmer, D. H., Obituary of Harry Schultz Vandiver, 1882-1973, 817

Lovelady, David Lowell. Corrigendum to: Bounded solutions of whole-line differential equations, 778

Meetings of the American Mathematical Society, Reports of September 1973, Rome Colloquium in Combinatorial Theory (Herbert S. Wilf), 238 August 1973, Missoula, summer meeting (Everett Pitcher and Kenneth A. Ross), 440 
October 1973, Cambridge (Walter Gottschalk), 443

November 1973, Minneapolis (Paul T. Bateman), 444

November 1973, Atlanta (O. G. Harrold), 445

November 1973, Tucson (Kenneth A. Ross), 651

January 1974, San Francisco, annual meeting (Everett Pitcher and Kenneth A. Ross), 652

March 1974, Gainesville (O. G. Harrold), 816

Membership, elections to, 42, 237, 439, 642, 808

Statement of Ownership, Management and Circulation, 1294

Treasurer's Report, 1101

Vandiver, Harry Schultz. See Lehmer, D. H.

Zieschang, Heiner. Addendum to: "On extensions of fundamental groups of surfaces and related groups", 366 